\title{
Vinculación de la investigación con las políticas educativas. El caso de Jalisco, México*
}

\section{Linking Research with Educational Policies: The Case of Jalisco State, México}

\author{
Víctor Manuel Ponce \\ grima**
}

Fecha de recepción: 29 de julio de 2011

Fecha de revisión: 12 de agosto de 2011

Fecha de aprobación: 26 de agosto de 2011

\section{Resumen}

Este trabajo aborda las estrategias que el Consejo Interinstitucional de Investigación Educativa (CIIE), de la Secretaría de Educación Jalisco (México), ha implementado para enfrentar el problema relativo a la debilidad de la vinculación de la investigación con las políticas educativas, problema reconocido ampliamente por los estudiosos del campo. En la primera parte de este trabajo se recogen los principales aportes de la investigación en torno a la explicación y las posibles soluciones al problema de la desvinculación entre investigación y políticas; luego se expone cómo se planteó el CIIE la solución a esa problemática. Posteriormente, se plantean las principales

Artículo de investigación científica y tecnológica, que presenta los resultados originales del proyecto terminado de investigación de la línea denominada «Procesos y prácticas de formación y políticas de investigación educativa».

** Doctor en Educación de la Universidad La Salle, México. Investigador de la Escuela Normal Superior de Jalisco y Secretario Técnico del Consejo Interinstitucional de Investigación Educativa de la Secretaría de Educación Jalisco. Investigador de las líneas de procesos y prácticas de formación y políticas de investigación educativa. 
estrategias desarrolladas. Por último, se hace un balance de esas experiencias y se proponen los desafíos para el futuro.

Palabras clave: políticas públicas, problemas de investigación, sistema educativo.

\begin{abstract}
This work exposes the strategies that the Interinstitutional Advice of Educational Investigation (IAEI), of the Secretary of Education of the State of Jalisco (México), has implemented to control the problem of the weakness of the relationship between research and educational policies. This problem is widely recognized by the experts. In the first part of this work we find the contributions by researchers on the explanation and solutions of the detachment problem between research and policies. Later the article approaches how the IAEI designed and implemented solutions to this problematic. Finally we reflect on the acquired experiences and future challenges are proposed.
\end{abstract}

Keywords: Public policies, research problems, educational system. 


\section{Introducción}

El propósito de este trabajo es debatir el problema de la debilidad de la vinculación de la investigación con las políticas educativas en nuestro país, problema reconocido internacionalmente por estudiosos del campo, por ejemplo, en los múltiples trabajos publicados por el COMIE (Consejo Mexicano de Investigación Educativa) y otros investigadores como Maggy (2003), Weiss (2004), Reimers y McGinn (2000), y Flores-Crespo (2008). También se sistematiza la experiencia del Consejo Interinstitucional de Investigación Educativa de la Secretaría de Educación Jalisco (CIIE), en torno a la intervención de esa problemática.

En los documentos de creación y en los programas del CIIE se estableció la urgencia de consolidar un modelo de investigación educativo, adecuado a las necesidades del desarrollo educativo del estado de Jalisco y de las políticas educativas de la Secretaría, que ponga a prueba dispositivos que promuevan su impacto en la toma de decisiones. El modelo de investigación propuesto considera de principal importancia la permanente comunicación entre investigadores y diversos agentes educativos, sobre todo, entre estos últimos, los responsables de la formulación e implementación de las políticas educativas estatales.

En la primera parte de este trabajo se plantean, desde los especialistas tanto en políticas como en la investigación educativa, las razones y posibles soluciones al problema de la desvinculación entre investigación y políticas, al igual que la necesidad de atenuar dicha problemáti- ca. En la segunda parte se expone el proyecto del CIIE, es decir, cómo se planteó la solución a ese problema. Posteriormente, se describen las principales estrategias desarrolladas con el propósito señalado. Al final, se hace un balance de esas experiencias y se proponen los desafíos hacia el futuro.

\section{El problema de la desvinculación de la investigación con las políticas}

Diversas investigaciones coinciden en señalar que Jalisco es uno de los estados con mayor producción de investigación educativa en el país (Sañudo y Ponce, 2003; Chavoya, 2006); sin embargo, otros estudios reconocen que esa fortaleza no se ha traducido en el desarrollo educativo de la entidad (Flores-Crespo, 2008; Kepowics y Santacruz, 2006).

Frente a esta circunstancia, Flores-Crespo (2008) cuestiona que el bajo desarrollo educativo puede deberse al problema de la desvinculación de la investigación con las políticas educativas, y aún más, que dicho problema rebasa al estado de Jalisco, incluso a nuestro país. Es un problema internacional, ampliamente documentado, por ejemplo, por Reimers y McGiin (2000) o por Latapí (2008).

Para los especialistas como Aguilar (2003), Parsons (2007) o Flores-Crespo (2008), es importante y urgente mejorar esa vinculación, pues el conocimiento especializado tiene una enorme importancia para identificar, definir con precisión e incluso proponer los problemas 
que se introducen a la agenda gubernamental, pero además para proponer y valorar los mejores cursos de acción para resolver dichos problemas.

Desde mi participación en los estados del conocimiento en el campo de la investigación educativa, producida en la última década del siglo pasado, se apuntaba el círculo vicioso entre el consumo y la producción de investigación, al menos para las unidades de investigación de la Secretaría de Educación Jalisco (SEJ), que se traduce en el poco apoyo financiero a la investigación, pero también en la desvinculación de la investigación para la toma de decisiones y las políticas educativas. Con respecto a lo anterior, Sañudo y Ponce (2003) afirman que:

Parece un círculo vicioso: no se apoya porque no se conoce la producción de investigación y no se conoce porque no se acerca la producción al usuario y no se acerca porque no se le apoya. Probablemente este círculo vicioso se pueda resolver en la medida en que los potenciales de la investigación se acerquen a las necesidades y prioridades de las dependencias, autoridades y los tomadores de decisión; lo cual puede conducir a la promoción de nuevos investigadores y al fortalecimiento de la calidad de la investigación. Seguramente que los círculos viciosos se pueden irrumpir desde diversas estrategias, como la búsqueda de mayor cercanía investigador-usuario, el apoyo a la formación de liderazgos académicos y el fortalecimiento de la cultura y la mayor calidad de la investigación (p. 428).

El problema es de vinculación, pero también de la naturaleza de los modelos y las condiciones que orientan tanto a la investigación como a la formulación e implementación de políticas.

Con respecto a la investigación, por ejemplo, una de las críticas de la Revisión nacional de investigación y desarrollo educativo sobre México, realizada por la OCDE-CERI (2004), señala que para los responsables de las políticas educativas nacionales, es difícil contar con buenos investigadores que realicen trabajos de investigación puntuales necesarios para tomar determinadas decisiones ${ }^{1}$.

Con respecto a las políticas, Messina (2006) argumenta que en realidad no existen verdaderas políticas "públicas" en América Latina, es decir, políticas discutidas "públicamente", porque no están insertas en debates públicos, en torno a la toma de decisiones acerca de qué problemas se incorporan a la agenda gubernamental y cuáles cursos de acción se seleccionan, sino que esta atribución ha quedado en manos de pocos agentes. En el caso de México, el campo ha sido dominado, de acuerdo con Ornelas (2009), por las autoridades de la Secretaría de Educación Pública (SEP) y sindicales, y que imponen a los estados sus políticas "centralistas".

Por otro lado, el desafío de transformar los sistemas de investigación educativa, los modelos para la formulación e implementación de políticas, así como de la vinculación entre ambos, es tanto una tarea gubernamental como de las instituciones responsables de la investigación y de la ciudadanía en general. Se trata de una

1 El CERI es un organismo dependiente de la OCDE, el cual realizó estudios en algunos países para evaluar el desarrollo del modelo de investigación para el desarrollo $(i+d)$ y su impacto en la educación básica. 
tarea orientada al desarrollo de sociedades democráticas, cuya gestión gubernamental debe ser por políticas verdaderamente públicas, es decir, transparentes en los argumentos que orientan las decisiones acerca de qué problemas se incorporan a la agenda gubernamental, cuáles cursos de acción se seleccionan, pero también transparentes en el ejercicio de los recursos humanos y financieros, gubernamentales, permanentemente limitados, para la solución de los problemas públicos.

Si bien los avances democráticos y el gobierno por políticas son notorios en las últimas dos décadas, no son pocos los que ponen en evidencia cómo las reglas del juego del ejercicio patrimonialista del gobierno no se han desmontado del todo, y se siguen ejerciendo como prebendas personales y partidistas de quienes gobiernan. En este sentido, Flores-Crespo (2008) provee argumentos para confiar en la posibilidad de la mejora de la vinculación de la investigación con las políticas, primero apoyado en la experiencia narrada por el propio Latapí (2008) como crítico externo, asesor y periodista, pero además, debido a las transformaciones del contexto macropolítico que están presionando a los gobiernos a regirse por políticas, así como el surgimiento de nuevos agentes y nuevos espacios de decisión, como el Consejo Nacional de Especialistas en Educación (CONAEDU), aún cuando su funcionamiento fue efímero. Sin embargo, reconoce la perseverancia de viejas prácticas antidemocráticas, autoritarias y patrimonialistas del ejercicio de gobierno en México.
Por otro lado, se han aportado diversas explicaciones para comprender la debilidad vinculatoria entre investigación y políticas educativas. Desde hace años, Latapí (1994) había señalado que el problema se debe a que los investigadores y los agentes de decisión poseen diferentes marcos de referencia, intereses, tiempos, lógicas, escalas de análisis e intereses.

También se ha señalado de manera reiterada que los tiempos de la investigación y la política son distintos; a los ritmos de la investigación se opone la urgencia de los funcionarios para la toma de decisiones y el logro inmediato de resultados (Maggi, 2003).

Además de las divergencias en la temporalidad, existen problemas de comunicación, así como de nivel y tipo de lenguaje empleado para comunicar los resultados de las investigaciones; pero se deben también a otras condiciones organizativas, culturales, institucionales, a la propia naturaleza de la comunidad educativa, e incluso a los modelos de investigación practicados en educación.

Los problemas culturales se refieren al hecho de que se trata de actores que pertenecen a "culturas" separadas, con intereses y lenguajes distintos, como lo refiere Latapí (2008). Aunque también existen factores de carácter institucional que obstaculizan la aplicación e impacto de los nuevos conocimientos, debido a la fuerza de la costumbre, a la inercia y a las culturas instituidas contrarias a lo que indican los resultados de la investigación (Guerrero, 2007). 
Otro nivel de problemáticas se localiza en los excesos burocráticos de los sistemas organizacionales y en las voluntades políticas de muchos agentes gubernamentales, que vuelven sumamente lento, incómodo y engorroso cualquier cambio significativo en las decisiones. Estos esquemas organizativos además impiden a los ejecutores contar con el tiempo suficiente para analizar y digerir cuerpos complejos de información.

En síntesis, los problemas de vinculación entre investigación y políticas tienen diversas explicaciones entre las que se han destacado las divergencias temporales, de esquemas culturales, intereses, códigos de entendimiento y comunicación, así como prácticas institucionales y organizativas, y la separación de las funciones entre investigadores y responsables de la hechura e implementación de políticas.

Se han sugerido diversas alternativas de solución para resolver la desvinculación entre investigación y políticas. Algunas se refieren a la transformación de los sistemas de investigación, otras a la transformación de los modelos de gobernanza, formulación e implementación de políticas. Otras se refieren a la transformación paralela de ambos subsistemas.

Las propuestas dirigidas hacia los sistemas de investigación se refieren a su fortalecimiento, pero también a la mejora de su calidad y pertinencia en la selección de los campos y líneas de investigación, orientados hacia los ámbitos emergentes de la política, sin que eso implique necesariamente que todo el sistema de investigación se vuelque en esa dirección.
Lo fundamental es que se invierta más en la investigación, pero desarrollada con mayor responsabilidad social (Reporte del CERI-OCDE, 2004; Maggi, 2003).

Desde el ámbito de las políticas, si bien existen fuerzas contrarias, es todavía poderoso el gobierno por políticas en el modo jerárquico burocrático, que ha fracasado reiteradamente en las políticas educativas de las últimas décadas del siglo pasado (Ornelas, 2009; Castillo y Asuma, 2009; Aguerrondo, 2002). En este modelo se confía en el "poder" del mandato de arriba-abajo, es decir, que las disposiciones de autoridad "bajan" y se ejecutan textualmente por cada uno de los estamentos de las jerarquías inferiores.

Frente a esos modelos de gobierno por mandato se oponen otros que enfatizan la importancia de la descentralización, al mismo tiempo que se otorga mayor autonomía a la escuela y la transformación del aparato del gobierno educativo, mediante la conformación de redes de políticas y la promoción de la colaboración entre las agencias participantes, desde la formulación hasta la implementación y seguimiento evaluativo de las políticas (Klijn, 1998; Goodin, Rein y Moran, 2006).

Para Goodin, Rein y Moran (2006), el acto de gobernar debería ser cada vez menos una tarea de mandatos de las autoridades jerárquicas y por el contrario, debería implicar cada vez más la negociación de procesos de descentralización, de alianzas colaborativas. Lo denominan como "network governance". En este modelo, los actores de los nodos centrales de estas 
redes no tienen la tarea de dictar las tareas que deben hacer los demás, sino de obtener una amplia cooperación de muchos actores independientes eficaces, pero orientados para que cada agencia cumpla sus objetivos.

Desde este contexto, para mejorar la vinculación entre investigación y políticas, son necesarias políticas formuladas e implementadas desde nuevos modelos participativos, pero también nuevas formas organizacionales y de gobierno, que promuevan la colaboración de diversas agencias, entre las que se encuentran los centros de investigación comprometidos, que proveen estudios de las problemáticas educativas prioritarias, de las estrategias, de los cursos de acción para su solución y en general de los procesos y los resultados de las políticas.

Por otro lado, conviene que los investigadores educativos no se muevan exclusivamente en los ámbitos académicos y al mismo tiempo, que los funcionarios no se muevan sólo en el ámbito de las organizaciones burocráticas. Para superar la dualidad investigación - política es urgente conformar espacios que promuevan intensas actividades de intercambio, entre las agencias de gobierno y de investigación. Goodin, Rein y Moran (2006) ofrecen argumentos sólidos en esa dirección, a través del establecimiento de redes de políticas, dentro de las cuales se incluyen a los especialistas e investigadores, al mismo tiempo que se cambian los estilos autoritarios de gobierno.

Uno de los dispositivos bastante conocido, dispuesto para asegurar la vinculación de los investigadores con los tomadores de decisio- nes son los diálogos informados. Se trata de una propuesta obtenida de la experiencia sistematizada de consultores internacionales de varios gobiernos en desarrollo. Los diálogos informados implican acciones encaminadas a intensificar la relación entre investigación y tomadores de decisión, es decir, para la vinculación de la investigación con la formulación y seguimiento de las políticas educativas (Reimers y McGinn, 2000).

Dentro de los modelos de Policies Network (redes de políticas) y de las políticas basadas en la evidencia de la investigación, Flores Crespo (2008) sugiere apoyar la iniciativa de systematic reviews, según la cual se busca propiciar el encuentro de investigadores con los tomadores de decisión, para poner en común el conocimiento en los contextos y situaciones con base en los cuales se toman las decisiones de políticas, y proveer debates públicos acerca de cuáles decisiones se apoyan en qué tipo de evidencias. Aunque también demanda que debe revisarse la calidad de la investigación y generar sistemas de comunicación transparente, tanto de la sistematización de los resultados de investigación como de las decisiones en lo referente a las políticas gubernamentales.

\section{Investigación que acompaña a las políticas. Estrategia del CIIE}

El Consejo Interinstitucional de Investigación Educativa (CIIE) fue fundado en 2004. El acuerdo que lo crea señala que su objeto es "promover y coordinar la implementación, operación, evaluación y reordenamiento de diversos planes, programas y acciones en materia de In- 
vestigación Educativa en la Secretaría de Educación Jalisco". Para cumplir ese propósito se plantearon tres objetivos: definir y orientar áreas y temas prioritarios de investigación; fomentar la investigación; y fortalecer los dispositivos para la diseminación, uso e impacto del conocimiento.

La revisión del estado de la cuestión acerca de los problemas en el uso y repercusiones de la investigación, y sobre todo de su vinculación con las políticas educativas, llevó al CIIE a la necesidad de constituirse en mediador, a través de la organización y diseño de modelos que faciliten el encuentro entre investigadores y funcionarios centrales y medios de la SEJ, como con sus asesores académicos o técnicos. Con el tiempo al modelo se le denominó "Investigación que acompaña a las políticas" (Ponce, 2009).

El modelo enfatiza en la necesidad de que el sistema de investigación estatal disponga de diversas interrelaciones con las políticas públicas en materia educativa. De acuerdo con Díaz Piña (2003) y Aguilar Villanueva (1994), las políticas públicas tienen cursos de acción que inician con su formulación y diseño, de lo cual se sigue su implementación, ajuste y evaluación. En este sentido, se planteó que la investigación podría ayudar a identificar los problemas de la agenda gubernamental, el diseño de políticas, y a retroalimentar los cursos de acción de las agencias de políticas durante su implementación, evaluación y ajuste, sin dejar de reconocer que la investigación debería estar alerta al reconocimiento de otros problemas que no están en la agenda pública para proponer nuevos asuntos prioritarios para la política.
Por lo anterior, desde el ámbito de la investigación, el sistema de acompañamiento a las políticas debería revisar el establecimiento de las prioridades de investigación, el proceso de producción de conocimiento, así como de los dispositivos para la diseminación, uso e impacto de la investigación educativa. El modelo debería promover investigaciones para monitorear el curso de acción de las principales políticas, así como la organización de encuentros periódicos entre investigadores y los equipos responsables de las políticas educativas. A través de estos encuentros se busca que los investigadores sean más sensibles a las necesidades de los tomadores de decisión; pero a su vez, los equipos responsables de las políticas y sus programas estratégicos, podrían retroalimentarse de los resultados de la investigación, para tomar mejores decisiones.

Por otro lado, se puede constatar el desarrollo de tendencias y cambios en los modos de producción de conocimientos internacionales, los cuales se distancian del modelo tradicional de investigación, de carácter disciplinar y muchas veces ajeno a las necesidades sociales del conocimiento. El nuevo modo de producción de conocimiento se origina en diversos lugares y no exclusivamente en la universidad, funciona dentro de un contexto de aplicación en el que los problemas no se encuadran dentro de una estructura disciplinar, sino que es inter y transdisciplinar; se organiza alrededor de problemas sociales y promueve una mayor responsabilidad social por el conocimiento. Por tanto, los criterios de evaluación del conocimiento no dependen de las comunidades científicas disci- 
plinares, sino que incluyen la valoración de los agentes participantes en el proceso investigativo y de los organismos públicos y privados, beneficiarios de la investigación (Gibbons y otros, 1997; Guillaumín, 2000).

Ese nuevo modo de producción de conocimiento, inter y transdisciplinar, vinculado a dispositivos de aplicación y relacionado con sistemas y grupos de profesionales del conocimiento, es cercano al modelo de "investigación que acompaña la política", pues prevé la posibilidad de que algunos agentes de políticas puedan participar en algunos momentos del desarrollo de la investigación, por ejemplo al incorporar nuevos conocimientos situados y complejos, de naturaleza interdisciplinar y transdisciplinar de los profesionales prácticos, que ayudan a visualizar mejor los problemas educativos, durante el diseño y desarrollo de la investigación. Evidentemente, estas investigaciones estarían dirigidas por investigadores experimentados.

El modelo de la investigación que acompaña a las políticas concibe a la investigación educativa como un sistema en el que se toman en cuenta diversos elementos, desde el diseño y operación de políticas que orienten la investigación hacia campos prioritarios, un proceso de producción dinámico y eficaz, la formación de investigadores especializados y comprometidos en los campos temáticos decididos, dispositivos pertinentes para el uso y aplicación del conocimiento para la mejora y el desarrollo educativo, pero además con recursos suficientes para su implementación.
En adelante se expone cómo se desarrollaron las actividades del CIIE, desde dos perspectivas: la primera perspectiva es longitudinal, pues recorre las principales etapas del CIIE, con el objeto de precisar cómo se relacionó el CIIE en cada uno de esos momentos decisivos para las políticas educativas estatales; en la segunda perspectiva, se explican las principales funciones y tareas emprendidas, con énfasis en las funciones como mediador o facilitador de dispositivos de comunicación entre investigadores y tomadores de decisión. Al final se propone un balance de las actividades, y algunos desafíos para el futuro.

Se pueden reconocer tres etapas del CIIE. La primera, de 2004 a 2006, va del nacimiento del CIIE al término del gobierno estatal en el sexenio anterior (2000-2006). En la segunda etapa, entre 2006 y 2007, se llevó a cabo la transición de los poderes del gobierno estatal anterior al actual. La tercera etapa corresponde al periodo actual, cuyo gobierno abarca del 2007 al 2013.

Etapa 1. Surge el CIIE prácticamente a la mitad del periodo gubernamental del sexenio anterior. Lo destacado de esta etapa es que el gobierno no pudo constituir políticas educativas estatales. Prácticamente asumía las políticas federales, con algunas, pocas, mediaciones estatales. En esta etapa el CIIE encuentra poca articulación con las autoridades centrales, pero una abundante interacción con los asesores técnicos de los mandos altos y medios, a través de seminarios académicos y de investigación educativa. 
En esta etapa se realizaron 32 seminarios de investigación en los que interactuaron investigadores con los asesores académicos de las áreas centrales y de los mandos medios de la SEJ. Se produjeron 275 ponencias que se pueden consultar en el portal de la SEJ/CIIE2. Los seminarios procuraban el diálogo entre investigadores y agentes educativos, la colaboración interinstitucional, el conocimiento de los principales debates investigativos, así como la discusión de los proyectos estratégicos y las necesidades de conocimiento de las diversas áreas de la Secretaría.

La participación en los seminarios fue abundante, contando con más de 400 personas de diversas dependencias de la SEJ. Destacan los posgrados, las unidades de la Universidad Pedagógica Nacional, las escuelas normales y las dependencias de educación básica. La colaboración de especialistas externos a la SEJ fue importante, no sólo por la cantidad de ponencias (26), sino sobre todo por la calidad de sus aportaciones.

Al inicio, durante la instalación de los seminarios surgieron resistencias. Los "prácticos" (asesores técnicos y mandos medios) concebían como arrogantes a los investigadores, al mismo tiempo que estos creían que los "prácticos" no podían aportar conocimiento alguno. Con el tiempo se reconoció que en los seminarios se ponían en juego diversos tipos de conocimiento discursivo; unos (asesores técnicos y mandos medios), sobre los complejos problemas prácticos, en ocasiones transdisciplinar pues

2 Disponible en <http://portalsej.jalisco.gob.mx/investigacioneducativa/>. rebasaba los marcos disciplinares, por ejemplo los obstáculos de un sistema burocratizado que enfatiza más el respeto a las jerarquías y las reglas formales que la cualidad de las prácticas profesionales de algunos agentes; otros, los investigadores y especialistas externos y de la propia SEJ, contribuían a la teorización o la conceptualización de problemas o situaciones, y la incorporación de nuevas formas de comprender los complejos problemas educativos. Lo más destacado de estos encuentros es que se fue avanzando en la conformación de espacios horizontales de diálogo. Todos aportan. Cabe señalar que el único requisito de participación era la elaboración de un trabajo escrito relativo al tema del seminario.

Tabla 1. Dependencias y Ponencias del Consejo Interinstitucional de Investigación Educativa (CIIE)

\begin{tabular}{|l|c|}
\hline \multicolumn{1}{|c|}{ Dependencias } & Ponencias \\
\hline Posgrados & 47 \\
\hline UPN & 44 \\
\hline Consejo de Investigación (CIIE) & 39 \\
\hline Escuelas normales & 29 \\
\hline Áreas de actualización & 17 \\
\hline Educación básica & 39 \\
\hline Educación permanente (adultos) & 5 \\
\hline Planeación & 3 \\
\hline CEMSSyT & 3 \\
\hline Organismos externos & 26 \\
\hline No refieren adscripción & 12 \\
\hline Textos integrados & 11 \\
\hline Total & 275 \\
\hline
\end{tabular}

Se eligió al seminario como formato del encuentro horizontal entre agentes educativos -principalmente asesores, formadores y mandos medios-y los investigadores externos y de la SEJ. 
La condición es que cada participante ponga a disposición de los demás sus aportaciones, sean avances o ensayos de investigación, experiencias sistematizadas o reflexiones desde la práctica. La continuidad de los seminarios estaba dirigida hacia la conformación de Redes Estatales. Ambos dispositivos pretendían además de la formación de los participantes, la diseminación de conocimientos distribuidos entre los académicos e investigadores, que por la estructura organizativa de la Secretaría, están aislados entre sí, y que de acuerdo con las experiencias internacionales pueden ayudar a fortalecer la conectividad de nuestros investigadores con los diversos agentes educativos. Las redes de investigación están organizadas con base en las áreas temáticas prioritarias de investigación educativa del CIIE, que se tenían en esa etapa.

1. En la red de políticas y reformas participaron investigadores, académicos y responsables de políticas educativas estatales y de las reformas educativas federales que se implementan en educación básica de la SEJ. Lo destacado de estos debates es la necesidad de desarrollar capacidades locales tanto para adaptar las políticas (homogéneas) de la federación (SEP) a las condiciones de Jalisco, como para promover políticas estatales, en el marco de las atribuciones estatales.

2. En la red de formación colaboran investigadores y asesores académicos de diversas instancias de formación de docentes, sobre todo de posgrados, normales y de actuali- zación. Se elaboraron diagnósticos y estados de la investigación sobre la educación normal, la actualización de docentes, los modelos de formación inicial y continua, y sobre los modelos de prácticas reflexivas.

3. La red de matemáticas permitió conectar diversos conocimientos parciales, generar nuevas investigaciones, sistematizar las experiencias acumuladas y difundir los resultados de las investigaciones. Destaca la participación de asesores de múltiples programas de la Secretaría, así como de investigadores, pero que afrontan la explicación y la solución de los bajos indicadores de los alumnos de educación básica desde perspectivas o enfoques distintos. Una tarea apuntada, que no fue desarrollada hasta el final, es la posibilidad de la articulación teórica y práctica de esa diversidad.

4. En la red de gestión educativa participaron tanto investigadores como asesores técnicos y mandos medios de educación básica. Coincidieron en algunas necesidades: 1) la formación académica de los directivos escolares, supervisores, asesores técnicos, indispensable para desarrollar mejor la gestión escolar; 2) el diseño de criterios generales para la definición de los perfiles normativos y académicos, que deben cubrir quienes asuman los nuevos cargos directivos para cada área o nivel específico, así como para la capacitación y formación de nuevos directivos; 3) la comprensión de modelos y teorías de gestión en el debate internacional, en contraste con los que se 
operan, de acuerdo con las concepciones, visiones y necesidades de cada nivel y área de la SEJ.

5. La red de práctica educativa fue de las más participativas. Colaboraron sobre todo investigadores, académicos, formadores y estudiantes de los posgrados educativos profesionalizantes. Los debates incluyen tanto la práctica docente y directiva en educación básica -como objeto de investigación-, como la práctica reflexiva -como estrategia formativa y de desarrollo profesional-. El primero se centra en los procesos de investigación, las metodologías, los marcos teóricos y conceptuales, así como los principales hallazgos de las investigaciones, locales y nacionales. El segundo trata de los procesos reflexivos acerca de la práctica. Conviene destacar que en este marco se promovieron cuatro investigaciones con el apoyo de Fondos Sectoriales del Consejo Nacional de Ciencia y Tecnología (CONACyT), sobre prácticas de educación especial, secundaria y educación indígena.

6. Atención educativa a los grupos socialmente vulnerables. La red involucró a investigadores, así como a los responsables de los programas compensatorios, indígenas y de educación especial. Acuerdan que la inequidad debe ser asunto prioritario de las políticas nacionales y estatales. Esta red partió del principio de que la política dirigida hacia la equidad, debe mejorar las variables de la oferta y la demanda educativas. Se reconoció que se invierten muchos recursos, y se operan diversos programas, pero que sin embargo, se implementan sin coordinación y, sobre todo, sin el acompañamiento de la investigación o la evaluación. Se plantearon diversas informaciones, aunque aisladas, que pueden conducir a la sospecha de que en la realidad no existen avances significativos en la superación de las condiciones de inequidad en que trabajan las escuelas rurales, indígenas y de las periferias urbanas.

7. En la red de procesos de aprendizaje y cognición se involucró sobre todo a investigadores y asesores técnicos de casi todos los niveles de la Secretaría. Se abordaron las teorías generales del aprendizaje y la cognición, y luego, específicamente la comprensión lectora y la enseñanza y aprendizaje de las ciencias. El aprendizaje constituye el motivo principal de la educación. Los seminarios documentaron discusiones locales e internacionales, sobre las teorías y prácticas que se mueven alrededor del aprendizaje. Acerca de los procesos de aprendizaje específicos, como la comprensión lectora, la resolución de problemas matemáticos y la enseñanza de las ciencias, se produce investigación de calidad en Jalisco, aunque menos sobre el aprendizaje de las ciencias.

8. Evaluación e indicadores educativos. En esta red participaron principalmente asesores y funcionarios sobre todo de las áreas de evaluación y de diversas dependencias de la SEJ. Los debates se refieren a la afirmación de que la conducción de los 
servicios educativos y del sistema educativo demanda de la evaluación, y para ello del diseño de criterios e indicadores de evaluación adecuados a la naturaleza de los propósitos educativos generales y específicos para cada área. En el medio se encuentran diversas conceptualizaciones, modelos de evaluación, criterios de construcción de indicadores, así como diversas evaluaciones que informan acerca del estado actual del sistema educativo, aportadas por estadísticas nacionales de la SEP, del Instituto Nacional de Evaluación Educativa y locales, estas últimas aportadas por las áreas de planeación y evaluación de la SEJ.

Las redes y los seminarios se desarrollaron incluso después de esta primera etapa, aunque con menos intensidad. En el CIIE, seguimos convencidos de que las redes y los seminarios se pueden constituir en dispositivos para la diseminación de conocimientos distribuidos entre los académicos e investigadores, que por la estructura organizativa de la Secretaría, están aislados entre sí (Ducoing, 2006).

Etapa 2. Transición en el cambio de gobernador (2006/2007). La realización de los seminarios y el desarrollo de las redes de investigación, aportó un conocimiento amplio de la estructura de la SEJ, las fortalezas investigativas y las necesidades de conocimiento de las principales áreas de la SEJ; sin embargo, a pesar de ello, el CIIE tuvo poca participación en el diseño de los programas de gobierno y en particular en el Programa Sectorial de Educación del gobierno actual (PSE). Conviene destacar este hecho como muestra de la debilidad del CIIE, en esa etapa crucial para la formulación de la agenda y de las políticas estatales.

Etapa 3. Lo destacado del gobierno actual es que tanto en el Plan Estatal de Desarrollo 2030 (PED 2030) como en el PSE, además del diagnóstico educativo estatal, se propuso atender ocho políticas estatales, a las se que denomina retos. Pero además, conformó sendos equipos interinstitucionales, con énfasis en la colaboración, para la atención de cada uno de los retos. En esos equipos participan los mandos y asesores responsables de las diversas áreas que tienen alguna injerencia en los procesos de implementación de esas políticas. Las políticas o retos enunciados originalmente son los siguientes:

1) Una administración más eficiente y ordenada del sistema educativo.

2) Mejoramiento de los indicadores educativos, con especial interés en la reprobación y deserción de educación secundaria y media superior.

3) Fortalecimiento de la cobertura en educación media superior y tecnológica.

4) Consolidación de la educación superior.

5) Abatir el analfabetismo y disminuir el rezago educativo.

6) Institucionalizar la formación, capacitación y actualización de supervisores, personal directivo y docente. 
7) Mejorar la enseñanza y propiciar el gusto por el aprendizaje de las matemáticas, ciencia e inglés, desde la educación básica.

8) Fortalecer la educación en valores.

Como puede notarse desde la enunciación de los temas, se trata de la atención de problemas educativos fundamentales para el desarrollo educativo del Estado, aunque esta selección de problemas, como toda agenda gubernamental, está acotada por los tiempos de entrega de los planes de gobierno, la limitación permanente de recursos y la fuerza del centralismo de las políticas federales.

El reto 1 se propone descentralizar poderes y facultades a las delegaciones regionales y a las escuelas, así como mejorar sus dispositivos de apoyo, desde la supervisión y la asesoría académicas. Esta idea es cercana a lo que sugieren Castillo y Azuma (2009) como la tercer ola de reformas educativas en América Latina.

El Programa Estatal de Desarrollo 2030 aclara que el logro de los retos implica necesariamente mejorar las capacidades de los agentes educativos, de los docentes, pero también de los directivos escolares, supervisores y jefes de sector. Ese es el reto 6 .

En los retos 2 y 7 se compromete a mejorar los graves indicadores educativos que se concentran sobre todo en las secundarias y preparatorias ubicadas en las regiones más apartadas y con menor índice de desarrollo humano de la entidad. A través de los retos 3 y 4 , se propone ampliar los grados de escolaridad de los jaliscienses y prever el cambio en las dinámicas poblacionales, que por un lado tienden a reducir las demandas de espacios en educación básica, y aumentar la demanda en educación media superior y superior. En el reto 5 se apremia a reducir de dos millones a un millón, la población mayor de 15 años que no ha culminado su educación básica. En todos estos casos, en términos generales, coincide con los indicadores nacionales educativos del Instituto Nacional de Evaluación Educativa en los cuales se confirman los problemas asumidos en la política estatal.

Finalmente, a lo largo del PED 2030 y del PSE se aduce que la práctica de los valores puede obstaculizar o facilitar el acto educativo, por eso es necesario fortalecer la educación de valores, en todos los espacios educativos y ámbitos de relación humana de la SEJ.

Conviene destacar dos fenómenos inéditos en el Estado. En primer lugar, la disposición de las autoridades gubernamentales y de la SEJ, de participar con sus políticas locales propias, y no depender sólo de los mandatos de la SEP, dentro de sus facultades legales. En segundo lugar, la conformación de equipos de trabajo por cada reto constituido, por los responsables de las áreas que tienen alguna responsabilidad en la atención de cada política. Su responsabilidad es la revisión de los diagnósticos del problema, así como de las estrategias que deben implementarse para lograr el reto. Esta estrategia 
de implementación de política se relaciona con las tendencias internacionales de Policies Network y las redes de colaboración, señaladas anteriormente.

No se está afirmando el éxito de las políticas educativas en Jalisco, pero es importante enfatizar lo que debe ser conservado, consolidado y profundizado, sobre todo en el contexto de la débil capacidad y experiencia de los Estados para gobernar sus propias políticas, del exceso del centralismo de la SEP y los pocos recursos financieros con los que cuenta. Por eso es sumamente importante documentar el caso, para dejar constancia de la experiencia, para que permanezca en las siguientes administraciones. También conviene advertir que estas formas de organización y de operación en equipos interinstitucionales de colaboración, para el ejercicio de las políticas estatales, deberían replicarse en todos los niveles de la estructura educativa. Esto no debe quedarse sólo en los órganos centrales de gobierno, sino que debe ampliarse y consolidarse, debe instituirse, pues las formas de gobierno jerárquicamente burocrático no se han desmontado del todo, y con ello la tentación autoritaria del gobierno por mandato. Se trata de tradiciones fuertemente arraigadas en nuestros sistemas educativos.

El modelo de "investigación que acompaña a la política" propuesto atrás tiene en esta etapa mejores posibilidades de implementación. Bajo estas condiciones, el CIIE acompañó durante 2009 a los ocho equipos de los retos o de las políticas estatales a sus reuniones quincenales, con diversos propósitos: proveer información organizada de productos de investigación, diseñar propuestas de investigación y sistematizar la experiencia de la conformación de los equipos de los retos para la atención de las políticas estatales.

\section{funciones $y$ oficios del ciie}

Anteriormente se señaló que el problema principal de la vinculación entre investigación y políticas se debe a las enormes diferencias de intereses, culturas, códigos de comunicación, espacios temporales y estructuras organizacionales e institucionales. Frente a eso, la principal función del CIIE ha sido mediar entre los investigadores y los funcionarios responsables de las políticas estatales. Hemos desplegado la capacidad para el diálogo horizontal y abierto entre ambos, la capacidad para traducir las demandas de conocimiento de los tomadores de decisión en problemas y objetos de investigación, para traducir resultados de investigación en posibilidades de uso y aplicación, y además para animar y fomentar la participación de investigadores del Estado en las problemáticas de conocimiento de la SEJ.

Con respecto a la escucha de las necesidades de las autoridades y sus asesores, y su traducción en prioridades o demandas de investigación, puede consultarse en el portal de la SEJ/CIIE, el Plan Maestro de Investigación Educativa (2005) y contrastarse contra las demandas de investigación para fondos mixtos 2006 y 2008, o las propuestas de investigación para cada reto educativo (2010). 
Por otro lado, no todo problema de conocimiento implica necesariamente realizar nuevas investigaciones. Algunas demandas de conocimiento están resueltas en los estados de la investigación. A partir de estos y otros recursos de información, el CIIE sistematizó información actualizada y se les proporcionó a los equipos de los retos 2, 3, 5, 6 y 8 .

Para el fomento de las demandas o prioridades de investigación, se promovieron cinco investigaciones de fondos sectoriales y tres investigaciones de fondos mixtos del CONACyT. Las producciones editoriales generadas a propósito de los fondos sectoriales fueron producidas por Vergara (2006), Villalpando (2006), Ledezma y García (2006), Zatarain Mendoza y Ponce (2006), y Ponce y Pérez (2006).

Las demandas de los fondos mixtos (2006) abordaron tres problemáticas: 1) las prácticas y los modelos educativos innovadores para las modalidades y niveles de educación básica, investigación coordinada por Luis Felipe Gómez del Instituto Tecnológico Superior de Occidente (ITESO), en la que participaron además investigadores de la Universidad de Guadalajara (UdeG), y de las unidades de Tlaquepaque y Guadalajara de la UdeG. María Luisa Chavoya, investigadora de la UdeG, coordinó el proyecto "Perspectiva de la oferta y demanda de los servicios de educación superior, para el período 2007-2025, de acuerdo con el desarrollo y crecimiento poblacional, social y económico del Estado de Jalisco". Por último, Roberto Sayavedra, del la Sociedad Mexicana para la
Divulgación de la Ciencia y la Tecnología (SOMEDICYT), coordinó el proyecto "Modelo de divulgación para fortalecer la cultura científica y tecnológica en el Estado". Estas investigaciones aportaron conocimientos para la atención de los retos de educación básica, educación media superior y superior (retos 2, 4 y 7).

A través del Concurso Estatal de Investigación Educativa se premiaron 14 investigaciones que a juicio de un Comité de Evaluación Externo aportaron conocimiento acerca de las problemáticas y políticas estatales.

Para apoyar la comunicación de los investigadores con los tomadores de decisión se realizaron diálogos informados, a propósito de los 10 textos de los Premios Estatales de Investigación Educativa y las 9 obras producidas por los Fondos de Investigación del CONACyT. Como ya se señaló atrás a partir de Reimers y McGinn (2000), se planteó la necesidad de establecer mediaciones que ayuden a comunicar los resultados de investigación con los tomadores de decisión, en este caso las autoridades educativas, asesores técnico-pedagógicos y formadores. 
Tabla 2. Libros publicados del Premio Estatal de Investigación Educativa

\begin{tabular}{|c|c|c|}
\hline Título & Autores & $\begin{array}{c}\text { Retos } \\
\text { relacionado }\end{array}$ \\
\hline $\begin{array}{l}\text { Análisis de congruencia } \\
\text { interna y externa de las } \\
\text { carreras que se imparten } \\
\text { en el Instituto Tecnológico } \\
\text { Superior de Puerto Vallarta }\end{array}$ & $\begin{array}{l}\text { Luis Roberto } \\
\text { Domínguez }\end{array}$ & 4 \\
\hline $\begin{array}{l}\text { Aprendiendo a estudiar } \\
\text { matemáticas mediante la } \\
\text { resolución de problemas }\end{array}$ & $\begin{array}{l}\text { Alejandro } \\
\text { Rodríguez } \\
\text { García }\end{array}$ & 2 y 7 \\
\hline $\begin{array}{l}\text { Escríbeme un cuento. } \\
\text { La alfabetización como } \\
\text { práctica social para } \\
\text { el desarrollo de las } \\
\text { competencias comunicativas }\end{array}$ & $\begin{array}{l}\text { Luz Eugenia } \\
\text { Aguilar } \\
\text { González }\end{array}$ & 2 y 7 \\
\hline $\begin{array}{l}\text { La práctica de la asesoría } \\
\text { en los procesos de } \\
\text { intervención de la MEIPE }\end{array}$ & \begin{tabular}{|l|} 
Hilda \\
Araceli Arias \\
Beltrán, \\
Mara Mayté \\
\end{tabular} & 6 \\
\hline $\begin{array}{l}\text { Los cursos de actualización } \\
\text { de los profesores y } \\
\text { su impacto en las } \\
\text { prácticas docentes del } \\
\text { nivel de Secundaria }\end{array}$ & $\begin{array}{l}\text { Margarita } \\
\text { Martínez } \\
\text { Camacho }\end{array}$ & 6 \\
\hline $\begin{array}{l}\text { Historia de un } \\
\text { pasado-presente } \\
\text { Las escuelas primarias en } \\
\text { Guadalajara (1867-1914) } \\
\end{array}$ & $\begin{array}{l}\text { María } \\
\text { Guadalupe } \\
\text { García } \\
\text { Alcaraz } \\
\end{array}$ & 2 y 7 \\
\hline $\begin{array}{l}\text { La subjetividad del director } \\
\text { escolar, un acercamiento a } \\
\text { sus imágenes e imaginarios }\end{array}$ & $\begin{array}{l}\text { Ana Cecilia } \\
\text { Valencia } \\
\text { Aguirre }\end{array}$ & 6 \\
\hline $\begin{array}{l}\text { La reinvención del } \\
\text { magisterio, entre el } \\
\text { anhelo y el poder. El } \\
\text { caso de los posgrados } \\
\text { magisteriales en Jalisco }\end{array}$ & $\begin{array}{l}\text { Sergio } \\
\text { Lorenzo } \\
\text { Sandoval } \\
\text { Aragón }\end{array}$ & 6 \\
\hline $\begin{array}{l}\text { Formación de educadores } \\
\text { holista, el desarrollo de las } \\
\text { habilidades docentes y sus } \\
\text { procesos de transferencia }\end{array}$ & $\begin{array}{l}\text { Gabriela } \\
\text { Flores } \\
\text { Talavera }\end{array}$ & 6 \\
\hline $\begin{array}{l}\text { Pedro la hace y Juan la } \\
\text { paga. La actualización } \\
\text { emergente de los docentes } \\
\text { de secundaria 1999-2005 }\end{array}$ & $\begin{array}{l}\text { Magdalena } \\
\text { Flores } \\
\text { Manríquez } \\
\text { y Felipe } \\
\text { Espinosa } \\
\text { Chávez }\end{array}$ & $1,2,6$ y 7 \\
\hline $\begin{array}{l}\text { La región centro de Jalisco. } \\
\text { Una mirada a la educación } \\
\text { media superior, propuesta a } \\
\text { partir de una investigación }\end{array}$ & $\begin{array}{l}\text { Micaela } \\
\text { Navarro } \\
\text { Paredes }\end{array}$ & $2 b$ y 3 \\
\hline
\end{tabular}

\section{Balance general y conclusiones}

En síntesis, como se planteó anteriormente, el modelo de "Investigación que acompaña las políticas" se facilitó en la última etapa del CIIE. Coincide con la conformación de ocho políticas o retos educativos y la organización de equipos de trabajo interinstitucional, responsables de cada política.

El CIIE ha promovido encuentros y seminarios para el diálogo y el intercambio, que facilitan la comunicación de investigadores y los tomadores de decisión. Sin embargo, el CIIE no ha logrado instituir el modelo de Investigación que acompaña las políticas, en primer lugar porque tampoco se ha instituido el gobierno por políticas -como se sabe ampliamente, las políticas y la experiencia en el gobierno por políticas, en modelos colaborativos, tienen el enorme riesgo de desaparecer en los momentos de cambios de autoridades gubernamentales-; en segundo lugar, porque tampoco se ha consolidado un sistema de estímulos que apoye el cambio en las reglas de juego y la mayor participación de los investigadores.

Desde el ámbito del CIIE, los riesgos residen en la debilidad de su presupuesto y de su personal comisionado de otras áreas de la SEJ. Con el cambio de autoridades, los comisionados tienen el riesgo de ser regresados a sus plazas de adscripción.

La vigencia de las funciones del CIIE fue más clara en el contexto de los 8 retos o políticas de la SEJ, al proveer conocimiento para la toma de decisiones, así como por las sugerencias que 
se generaron para la conducción de la política educativa estatal. La mayoría de los equipos de los retos asumieron públicamente que el CIIE les aportó conocimiento para la toma de decisiones. Se ha probado que el modelo de la investigación que acompaña a la política si funciona, pero necesita condiciones para su eficacia y sobre todo para su consolidación institucional.

Por último, los desafíos que se deben enfrentar en el futuro inmediato, con el propósito de avanzar en la consolidación del CIIE y la vinculación de la investigación con las políticas son los siguientes:

1. Deben consolidarse los aprendizajes del gobierno por políticas educativas locales. Para ello, se está sistematizando la experiencia del trabajo de los equipos de los retos. Con este producto, se tienen previstas diversas reuniones de diálogos informados y seminarios con los equipos de los retos de la SEJ.

2. Dejar constancia de la experiencia del CIIE, para avanzar y no retroceder. Se debe fortalecer la capacidad técnica del CIIE en todos los ámbitos, en particular en los dispositivos para el fomento, diseminación, uso e impacto de los conocimientos generados por la investigación educativa. Para ello, debe operar con presupuesto suficiente.

3. Debe mejorarse aún más el sistema estatal de investigación. Las necesidades de conocimiento son muchas y la capacidad para producir, aunque sólida, sigue siendo débil (Congreso Estatal de Investigación para la Educación Básica, ver página en internet del CIIE). También deben actualizarse las prioridades de investigación, vinculadas al seguimiento de las políticas educativas y de las necesidades del desarrollo institucional y organizacional, así como prácticoprofesionales de los agentes educativos.

Hacen falta muchas cosas por hacer. Seguimos arando.

\section{Referencias}

Aguerrondo, I. (2002). Ministerios de Educación: de la estructura jerárquica a la organización sistémica en red. UNESCO: Instituto Internacional de Planeamiento de la Educación. IIPE/UNESCO. Sede Regional Buenos Aires.

Aguilar, L. E. (2008). Escríbeme un cuento. La alfabetización como práctica social para el desarrollo de las competencias comunicativas. Jalisco, Guadalajara: Secretaría de Educación.

Aguilar Villanueva, L. (1994). La Hechura de las políticas (Segunda Antología). México: Porrúa.

Aguilar Villanueva, L. (2003). La implementación de las Políticas. México: Porrúa. 
Aguilar Villanueva, L. (2007). Problemas públicos y agenda de gobierno (Colección Antologías de Política Pública. Primera Antología). México: Porrúa.

Arias, H. y Martínez, M. (2008). La práctica de la asesoría en los procesos de intervención de la MEIPE. Jalisco, Guadalajara: Secretaría de Educación.

Castillo, G. y Azuma, A. (2009). Las reformas y las políticas educativas. Impacto en la supervisión escolar. México: FLACSO.

Chavoya Peña, M. L. (2006). "Reflexiones sobre la práctica de investigar en educación", Revista La Tarea. Sección 47 del SNTE. No. 19, Guadalajara, Jalisco.

Díaz Piña, A. (2003). Las Políticas Públicas en Materia Educativa. México: Secretaría de Educación Pública.

Domínguez, L.R. (2008). Análisis de congruencia interna y externa de las carreras que se imparten en el Instituto Tecnológico Superior de Puerto Vallarta. Jalisco, Guadalajara: Secretaría de Educación.

Ducoing Watty, P. (Coord.). (2006). Sujetos, actores y procesos de formación. (Colección "La investigación educativa en México 1992-2002. Tomo II). México, D.F.: IPN - COMIE.
Espinosa, F. y Flores, M. (2010). Pedro la hace y Juan la paga. La actualización emergente de los docentes de secundaria 1999-2005. Jalisco, Guadalajara: Secretaría de Educación.

Flores-Crespo, P. (2008). Investigación educativa y políticas públicas en México: una relación amorfa y elusiva, Revista Sinéctica, 33. Guadalajara, Jalisco: ITESO.

Flores Talavera, G. (2010). Formación de Educadores holista, el desarrollo de las habilidades docentes y sus procesos de transferencia. Jalisco, Guadalajara: Secretaría de Educación.

García, G. (2008). Historia de un pasadopresente. Las escuelas primarias en Guadalajara (1867-1914). Jalisco, Guadalajara: Secretaría de Educación.

Gibbons, M. y otros. (1997). La nueva producción del conocimiento. Barcelona: Palomares-Corredor.

Goodin, R., Rein, M. \& Moran, M. (2006). The Oxford Handbooks of Political Science. Oxford: Oxford University Press.

Guerrero, L. (2007). Siete razones para tomar decisiones sin información. Disponible en http://educhevere.blogspot.com/2007/05/ siete-razones-para-tomar-decisiones-sin. html 
Guillaumin, A. (2000). Complejidad, transdisciplinariedad y redes: hacia la construcción de una nueva universidad. $\mathrm{CEIICH} \mathrm{-} \mathrm{Uni-}$ versidad Nacional Autónoma de México.

Instituto Nacional para la Evaluación de la Educación.(2008). El aprendizaje en tercero de secundaria en México. Informe sobre los resultados del Excale 09, aplicación 2008. México: Editorial del Instituto Nacional.

Kepowics, B. y Santacruz, L. (2006). "Región Centro-Occidente”. En Rueda, M. (coord.). Notas para una agenda de investigación educativa regional. México: COMIE.

Klijn, E. (1998). "Policy Networks: An Overview", En Kickert, W.J.M. \& Koppenjan, J.F. (Eds.). Managing Complex Networks. London: Sage. Disponible en http://revista-redes. rediris.es/webredes/textos/Complex.pdf

Latapí, P. (1994). La investigación educativa en México. México: Fondo de Cultura Económica.

Latapí, P. (2008). ¿Pueden los investigadores influir en la política educativa?. Disponible en http://www.oei.es/noticias/spip. php?article3534

Ledezma, M. y García, L. (2006). Una propuesta de intervención como apoyo a los docentes de matemáticas en una escuela secundaria. Guadalajara: Secretaría de Educación Jalisco.

Maggy, R. (2003). "Usos e impactos de la investigación educativa". En Weiss, E. (coord.). El campo de la Investigación educativa. La investigación educativa en México 19922002. México: COMIE/CESU-UNAM.

Martínez, M. (2008). Los cursos de actualización de los profesores y su impacto en las prácticas docentes del nivel de Secundaria. Guadalajara: Secretaría de Educación Jalisco.

Messina, G. (2006). “El lugar de la investigación en la educación básica", En Memorias del encuentro "Educación y organizaciones sociales: reflexiones desde la investigación y la práctica”. México, Universidad Iberoamericana. Disponible en http://www.uia. $\mathrm{mx} /$ web/site/tpl-Nivel2.php?menu=mglnv estigacion\&seccion=invInidelineas_etpanteriores2

Navarro, M. (2010). Vinculación del currículo con el mercado laboral en la educación media superior de sostenimiento estatal de la región centro de Jalisco. Jalisco, Guadalajara: Secretaría de Educación.

OCDE-CERI. (2004). Revisión Nacional de Investigación y Desarrollo Educativos. Reporte de los examinadores sobre Méxi- 
co. En Revista Mexicana de Investigación Educativa, No. 21, Vol. IX.

Ornelas, C. (2009). Política, poder y pupitres. Crítica al "nuevo" federalismo educativo. México: Editorial Siglo XXI.

Parsons, W. (2007). Políticas públicas: una introducción a la teoría y la práctica del análisis de políticas públicas. México: FLACSO.

Ponce Grima, V. y Pérez, V. (2006). La práctica y los significativos educativos de los agentes de los centros de atención múltiple que ofrecen educación primaria en el estado de Jalisco. Jalisco, Guadalajara: Secretaría de Educación.

Ponce Grima, V. (2009). Investigación y políticas educativas. En Revista Electrónica Sinéctica, 33. Instituto Tecnológico y de Estudios Superiores de Occidente, México.

Reimers, F. y McGinn, N. (2000). Diálogos informados. El uso de la investigación para conformar la política educativa. México: CEE/AUSJAL.

Rodríguez García, A. (2008). Aprendiendo a estudiar matemáticas mediante la resolución de problemas. Jalisco, Guadalajara: Secretaría de Educación.
Sandoval, S. (2010). La reinvención del magisterio, entre el anhelo y el poder. El caso de los posgrados magisteriales en Jalisco. Jalisco, Guadalajara: Secretaría de Educación.

Sañudo, L., Ponce Grima, V. (coord.). (2003).

"La investigación educativa en Jalisco. 1993-2001". En Weiss, E. (coord.). El campo de la Investigación educativa. La investigación educativa en México 19922002. México: COMIE/CESU-UNAM.

Tedesco, J.C. (2008). ¿Son posibles las políticas de subjetividad? En Tenti Fanfani, E. (comp.). Nuevos temas en la agenda de política educativa. Buenos Aires: UNESCO - IIPE - Siglo XXI editores.

Valencia, A.C. (2010). La subjetividad del director escolar, un acercamiento a sus imágenes e imaginarios. Jalisco, Guadalajara: Secretaría de Educación.

Vergara, M. (2006). Condiciones y Mejoramiento de la Educación Intercultural de los Estudiantes Indígenas, Nahuas y Huicholes de Educación Primaria en Jalisco. Jalisco, Guadalajara: Secretaría de Educación.

Villalpando, M. (2006). El Impacto de los Cursos de Actualización en el Desarrollo Profesional del Docente de Educación Básica. Jalisco, Guadalajara: Secretaría de Educación. 
Weiss, E. (2004). La gestión de la investigación educativa, En Revista Mexicana de Investigación Educativa, 21 (IX).

Zatarain, R. y Ponce, V. (2006). Gestión y Accio- nes Educativas en el Marco de la Reforma Integral de la Escuela Secundaria. México: Editorial Endora. 